\title{
Spectra of solar energetic particles and galactic cosmic rays over a million years reconstructed using aluminium-26 data from lunar rocks
}

\author{
Stepan V. Poluianov* \\ Space Climate Research Unit, University of Oulu, Finland, \\ Sodankylä Geophysical Observatory, University of Oulu, Finland \\ E-mail: stepan.poluianov@oulu.fi
}

\section{Gennady A. Kovaltsov}

Ioffe Physical-Technical Institute, St. Petersburg, Russia

\section{Ilya G. Usoskin}

Space Climate Research Unit, University of Oulu, Finland,

Sodankylä Geophysical Observatory, University of Oulu, Finland

\begin{abstract}
Direct measurements of solar energetic particles (SEP) cover the space era of several decades, but indirectly they can be studied for thousands and millions of years backward using cosmogenic nuclides in lunar rocks and soil. With a proper nuclide production model, it is possible to estimate the mean energy spectrum of SEP, as well as of galactic cosmic rays (GCR) from a depth profile of the measured nuclide content.

Here we used aluminium-26 (lifetime 1.03 Myr) measurements in Apollo-mission lunar samples. Previous estimates of the SEP spectrum from lunar data were based on the assumed specific shape and only provided reconstructed spectral parameters. We report a different approach to use a lunar rock as an integral spectrometer within $20-80 \mathrm{MeV}$. With that, one can reconstruct the particle spectrum directly without any a-priori assumptions on its exact shape.

For each studied lunar sample, we have developed an accurate Geant 4 model. We estimated the average GCR spectrum over the last million years (the modulation potential $496 \pm 40 \mathrm{MV}$ ), which is consistent with that for the Holocene ( $449 \pm 70 \mathrm{MV}$ ), but significantly lower than that for the modern epoch $(660 \pm 20 \mathrm{MV})$. We also made a true reconstruction of the mean SEP spectrum over the last million years. The integral flux $>30 \mathrm{MeV}$ was estimated as 37.4 particles $/\left(\mathrm{cm}^{2} \mathrm{~s}\right)$, which is consistent with that for the modern epoch. The estimated occurrence probability of SEP events shows no expected events with fluence $>30 \mathrm{MeV}$ over $5 \times 10^{10}$ and $1 \times 10^{11}$ particles $/ \mathrm{cm}^{2}$ on millennial and Mega-year time scales, respectively.
\end{abstract}

36th International Cosmic Ray Conference -ICRC2019-

July 24th - August 1st, 2019

Madison, WI, U.S.A.

* Speaker. 


\section{Introduction}

Solar energetic particles (SEP) make a very important contribution into the radiation environment in the near-Earth's space. Direct measurements of SEP are available only for several decades of the space era. For longer time scales, terrestrial natural archives of cosmogenic nuclides (e.g., tree rings, ice cores) can provide only very limited information, especially about weak/moderate SEP with energies about a few tens of MeV. Fortunately, lunar samples returned to the Earth, contain useful records about them in the from of depth profiles of in situ produced cosmogenic nuclides.

This idea to study SEP with those data was explored earlier (e.g., Reedy and Arnold, 1972; Fink et al., 1998; Jull et al., 1998; Nishiizumi et al., 2009, and many others). However, previous works modelled the depth profile as a function of the SEP spectral model, and fitted it to measured data, thus obtaining spectral parameters. This means that the SEP spectrum was prescribed by an assumed model with given shape. In this work, we use ${ }^{26} \mathrm{Al}$ produced in situ in lunar rocks and show that it can be utilised as a particle integral spectrometer and the mean SEP spectrum can be reconstructed directly from measurements point by point without any a priori assumption on its shape.

\section{Isotope measurements and model}

This work is based on cosmogenic nuclide ${ }^{26} \mathrm{Al}$ with the lifetime of $1.03 \mathrm{Myr}$, which was measured in lunar samples and soil. In particular, we used two samples 64455 and 74275 brought by Apollo missions 16 and 17, respectively. To evaluate galactic cosmic rays (GCR), we also used ${ }^{26} \mathrm{Al}$ data from Apollo-15 deep drill core. Rock 64455 has an ellipsoid-like form and is about 5 $\mathrm{cm}$ long and $3 \mathrm{~cm}$ across. The exposure age and erosion rate were estimated as $2 \mathrm{Myr}$ and $0-0.5$ $\mathrm{mm} / \mathrm{Myr}$, respectively. The content of ${ }^{26} \mathrm{Al}$ was measured by Nishiizumi et al. (2009). Sample 74275 is knob-like, $17 \mathrm{~cm}$ long, $12 \mathrm{~cm}$ across and $4 \mathrm{~cm}$ thick.The exposure age and erosion rate were estimated as $2.8 \mathrm{Myr}$ and $1-2 \mathrm{~mm} / \mathrm{Myr}$, respectively. Measurements of ${ }^{26} \mathrm{Al}$ were conducted by Fink et al. (1998). The drill core is $242 \mathrm{~cm}$ long and it provides a depth profile down to about $390 \mathrm{~g} / \mathrm{cm}^{2}$. Aluminium-26 was measured by two groups Rancitelli et al. (1975) and Nishiizumi et al. (1984) there. Physical and chemical properties of these lunar samples can be found elsewhere (e.g., Meyer, 2012).

The model of nuclide production uses the yield function approach, which separates the energetic particle spectrum and yield of nuclide of interest. The details of applications and related computations are described elsewhere (e.g., Poluianov et al., 2016). Yield functions have been computed for each sample individually. To evaluate nuclide production by GCR in the Apollo- 15 core, we used Monte Carlo simulations with Geant4 (Agostinelli et al., 2003). For SEP in samples 64455 and 74275 , we chose the analytical approach without cascades that works reasonably well for depths $<7 \mathrm{~g} / \mathrm{cm}^{2}$. The rocks were considered as laying on top of the lunar surface represented by data from the Apollo- 15 drill. A yield function is strongly dependent on the cross-section data for nuclear reactions leading to production of a nuclide of interest. In this work, we used ${ }^{26} \mathrm{Al}$ cross-sections from (Reedy, 2007; Nishiizumi et al., 2009; Reedy, 2013). Production by pions was also taken into account (Li et al., 2017). ${ }^{26} \mathrm{Al}$ has relatively low energy threshold and high 
efficiency of production. This makes it more preferable to study low energy particles such as SEP comparing to ${ }^{14} \mathrm{C},{ }^{10} \mathrm{Be},{ }^{36} \mathrm{Cl}$ and other nuclides measured in lunar samples.

The yield of ${ }^{26} \mathrm{Al}$ as a function of energy has a step-like shape at shallow layers $\left(<7 \mathrm{~g} / \mathrm{cm}^{2}\right)$ (Figure 1). It has a sharp energy threshold, which gradually increases with depth, and is more or less flat above that. Thus, the amount of produced nuclide atoms can be directly related to the integral particle flux $F(>E)$. The threshold is defined by particular spallation reactions on main targets in lunar material. At low depths under a few tens $\mathrm{g} / \mathrm{cm}^{2}$, there is direct production by incident energetic particles without developing of a cascade. The ${ }^{26} \mathrm{Al}$ yield function for lunar samples looks like one of an ideal integral spectrometer, which response is linearly related to the integral flux $F(>E)$ of incident particles. Therefore, it can be possible to reconstruct $F(>E)$ from measured ${ }^{26} \mathrm{Al}$ point by point, not by fitting a theoretical depth profile corresponding to an assumed $F(>E)$ model.



Figure 1: Yield function of ${ }^{26} \mathrm{Al}$ by protons. Yield is shown in arbitrary units here.

Effective energy $E^{*}$ and conversion coefficient $K$ are two main parameters needed to compute the integral flux $F\left(E^{*}\right)$ of incident particles from measured data by the described here method. The effective energy is defined in the same way as by Kovaltsov et al. (2014). It is the energy, for which conversion coefficient $K=F\left(>E^{*}\right) / A$ is most independent on spectral parameters (described below). Here $A$ is the activity of ${ }^{26} \mathrm{Al}$ in a studied sample, which can be measured or computed from theoretical production rate $Q$ as $A(h)=\int_{0}^{T} Q\left(h^{\prime}\right) e^{-t / \tau} d t$, where $h$ is the depth of a studied sample, $h^{\prime}=h+\rho r t$ is the depth $\left(\mathrm{g} / \mathrm{cm}^{2}\right)$ at time $t(\mathrm{Myr})$ taking into account erosion with rate $r(\mathrm{~mm} / \mathrm{Myr})$, density $\rho\left(\mathrm{g} / \mathrm{cm}^{2}\right) ; \tau$ is the lifetime of our isotope; $T$ is the sample exposure age.

With conversion coefficient $K(h)$, one can directly translate the measured activity $A_{\text {meas }}(h)$ of a nuclide of interest at depth point $h$ to the integral flux of incident particles $F\left(>E^{*}\right)$ as

$$
F\left(>E^{*}\right)=K(h) A_{\text {meas }}(h) .
$$

To estimate $K(h)$ and $E^{*}(h)$, which are individual for every sample, we had to compute theoretical ${ }^{26} \mathrm{Al}$ activity $A_{\text {model }}$ under incident particle flux $F(>E)$. For that purpose, we considered two widely used models of the SEP spectrum: the exponent over rigidity $R$ (EXP) and the power 
law over energy $E$ (POW),

$$
F(>R)=F_{0} e^{-R / R_{0}} \text { and } F(>E)=F_{0} E^{-\gamma},
$$

respectively, where $R_{0}$ and $\gamma$ are spectral parameters of those models that define the hardness. POW tends to provide a too hard spectrum, while EXP is too soft. There is also a number of other more realistic models laying between these two cases. Thus, EXP and POW can be considered as reasonable boundaries of more realistic scenarios. At a given depth, theoretical activity $A_{\text {model }}$ was calculated with a corresponding yield function for EXP and POW over a wide range of parameters $R_{0}$ and $\gamma$ resulting to a range of $F(>E)$. Then, we computed $K\left(E^{*}\right)=F\left(>E^{*}\right) / A$. Coefficient $K$ varies with the spectral parameter and $E^{*}$, but it is possible to find such $E^{*}$, for which the coefficient $K$ is almost independent on $R_{0}$ or $\gamma$. This defines the pair of $K$ and $E^{*}$ for a given sample depth $h$.

The computed conversion coefficient $K$ and effective energy $E^{*}$ for sample 64455 are shown in Figure 2. They are similar to ones for sample 74275. One can see that the EXP and POW curves of $E^{*}(h)$ are almost identical from the surface to at least $5 \mathrm{~g} / \mathrm{cm}^{2}$. Conversion coefficients $K(h)$ are also close to each other within $0.1-5 \mathrm{~g} / \mathrm{cm}^{2}$, but diverge outside this range. This defines the energy range of applicability of the method as $20-80 \mathrm{MeV}$ (from $E^{*}(h)$ ).
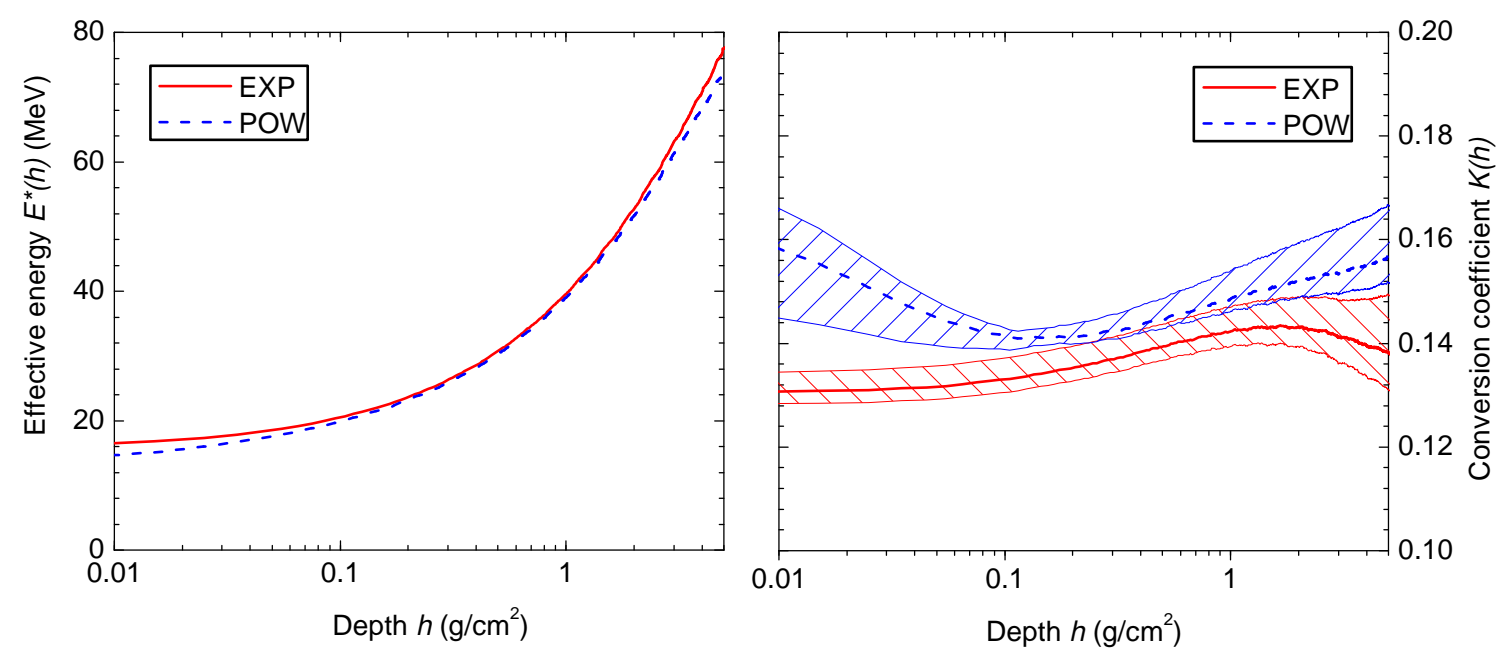

Figure 2: Effective energy $E^{*}$ and conversion coefficient $K$ as functions of depth $h$ computed for SEP spectral models EXP and POW and lunar sample 64455. The hatched areas indicate uncertainties.

Lunar surface is bombarded by both SEP and GCR. To estimate the nuclide production by solar particles, the GCR background should be subtracted. We used the force-field approximation of the GCR spectrum at the Earth's orbit, which works reasonably well in application to cosmogenic nuclide production. In this approach, we defined the GCR local interstellar spectrum (LIS) according to Vos and Potgieter (2015). Heliospheric modulation of LIS is represented by a single parameter called the modulation potential $\phi$ corresponding to some level of solar activity. The method provides the differential intensity spectrum for GCR protons, helium and heavier ions. The result used with the computed ${ }^{26} \mathrm{Al}$ yield function gives the nuclide production rate over a grid of depths. In particular, this can be computed as a function of $\phi$ for further fitting into experimental data. 


\section{Results}

To evaluate the SEP-produced ${ }^{26} \mathrm{Al}$ in samples 64455 and 74275 , we had to subtract the amount of that nuclide produced by GCR. For that purpose, we used the same ${ }^{26} \mathrm{Al}$ model and data from the deep core acquired by the Apollo-15 mission. It provides the nuclide depth profile beyond the depth of $20 \mathrm{~g} / \mathrm{cm}^{2}$, where all ${ }^{26} \mathrm{Al}$ is produced solely by GCR. We used these data to fit our GCR-produced nuclide depth curve and estimated the mean heliospheric modulation $\phi$ on the Myr time scale. Aluminium-26 measured in the core, as well as our model are shown in Figure 3. The best $\chi^{2}$-fit is provided by the mean heliospheric modulation potential $\phi=496 \pm 40 \mathrm{MV}$. This value is consistent with $449 \pm 70 \mathrm{MV}$ for the last 11000 years of the Holocene (reduced to the same LIS, Usoskin et al. (2016)), but is notably lower than one for the modern epoch (660 $\pm 20 \mathrm{MV}$ for 1951-2016, Usoskin, 2017). The defined $\phi$ is used for estimate of the background ${ }^{26} \mathrm{Al}$ produced by GCR.

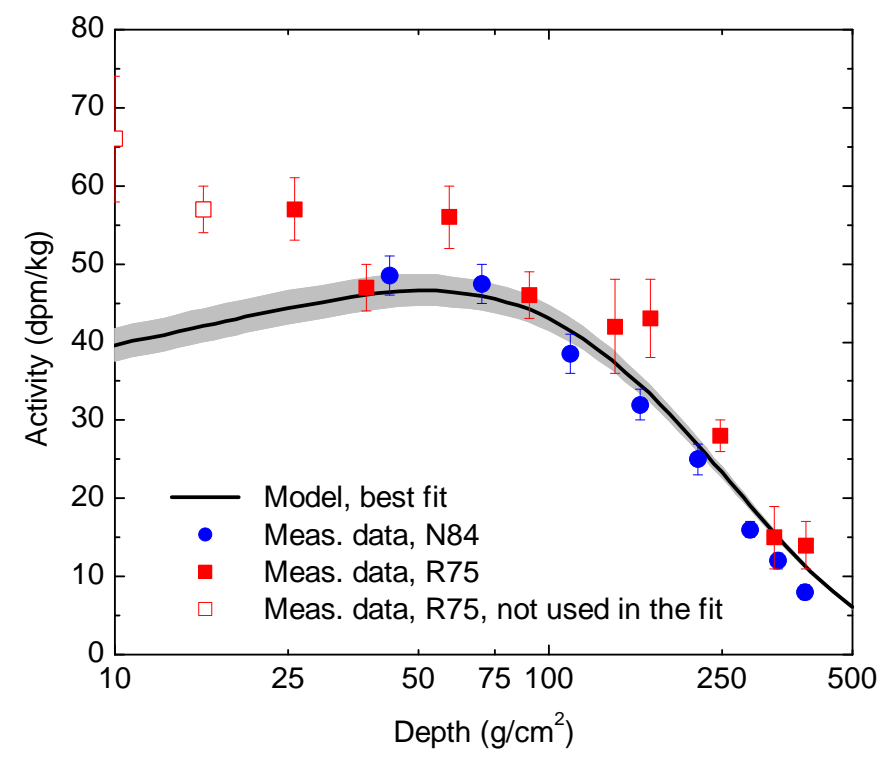

Figure 3: Measurements and model of ${ }^{26} \mathrm{Al}$ produced by GCR in lunar soil. The best model fit corresponds to $\phi=496 \pm 40 \mathrm{MV}$, the grey area represents $1-\sigma$ errors. In the legend, R75 is measurements from Rancitelli et al. (1975), N84 is ones from Nishiizumi et al. (1984). Measurements $<20 \mathrm{~g} / \mathrm{cm}^{2}$ were not used for the fit.

After subtraction of the GCR background in samples 64455 and 74275, the remained activity data were ascribed to SEP. The mean SEP spectrum was reconstructed according to Eq. 2.1. The results for two samples and different erosion rates, as well as their mean, are shown in Figure 4. The reconstructed points lay close to each other at energies 20-35 MeV regardless of their sample origin and erosion assumption. The uncertainty in that range is within $20 \%$. However, it grows with energy and reaches a factor of two at $80 \mathrm{MeV}$.

Comparison of the calculated SEP flux with earlier works (Fink et al., 1998; Jull et al., 1998; Nishiizumi et al., 2009) is shown in the right panel of the figure. They all agree with a factor of two and lay within the uncertainty of the present reconstruction. The estimate of SEP flux by Reedy (2012) for resent solar cycles 1954-2008 from space-borne measurements is also shown as triangles there. Although being observed in the period of the Modern Grand Maximum, it agrees well with the Myr-long mean result of this work. This fact shows no sign of a notable dependence of the mean SEP flux on the overall solar activity level. 

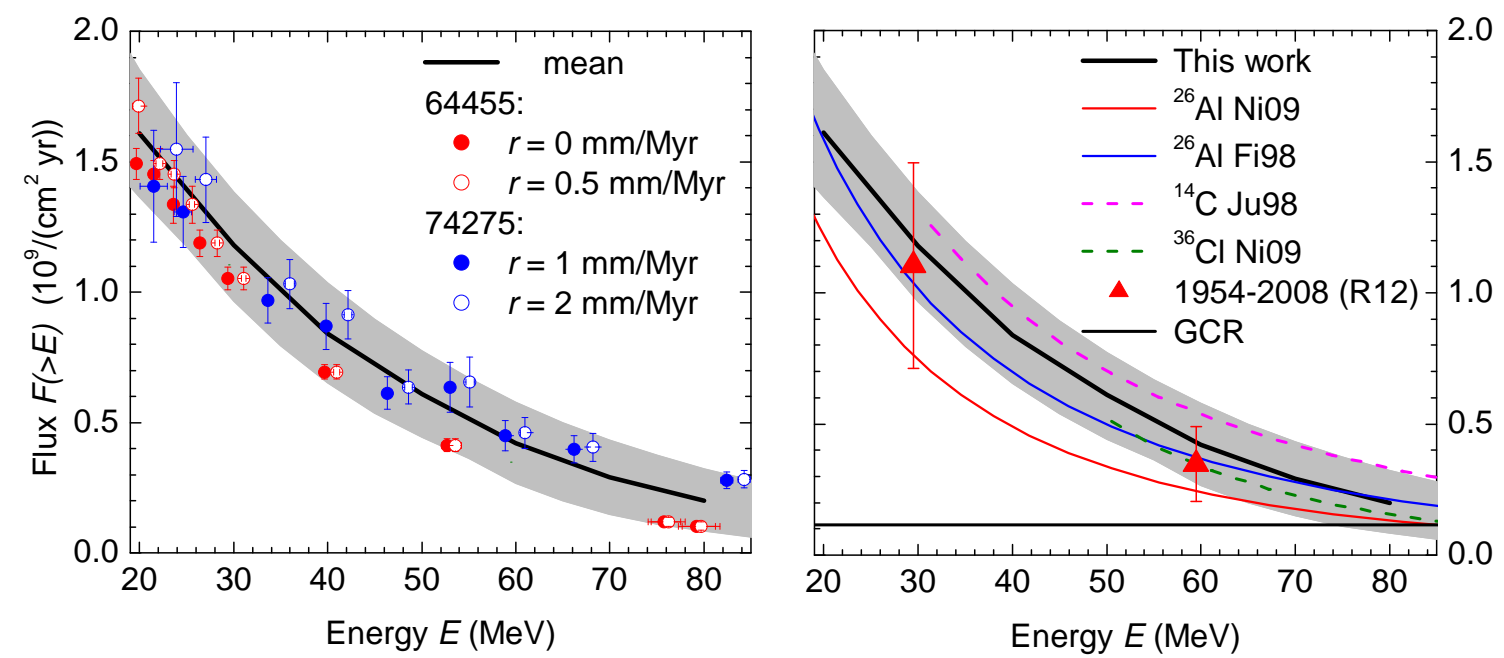

Figure 4: SEP integral omnidirectional flux $F(>E)$. Left panel: reconstruction of the mean spectrum on the Myr time scale from ${ }^{26} \mathrm{Al}$ with the method described in this work. The black line is the mean reconstruction from both samples 64455 and 74275, the grey area indicate its full-range uncertainties. The circle marks represent individual reconstructions from those samples for different erosion rates (see the legend). Right panel: Comparison of the result from this work with other spectra. The black line is the same as in the left panel. In the legend, Ni09 is Nishiizumi et al. (2009), Fi98 is Fink et al. (1998) and Ju98 is Jull et al. (1998). The triangles indicate the results for the modern space era (R12 means Reedy (2012)). The thin black line at the bottom represents the mean GCR for comparison.

We also computed the occurrence probability distribution of SEP events as a function of the annual fluence $F_{30}$. It is approximated by the Weibull distribution often used in such cases. The model was fitted to the data with conditions including ones from historical strong SEP events, upper limits on SEP events within the Holocene, and some others. The SEP probability distribution from this work is shown in Figure 5 as a hatched area along with estimates from space-borne data and terrestrial nuclide archives. The computed probability distribution agrees well those data except for one point from terrestrial isotopes at $F_{30}=2 \times 10^{9}\left(\mathrm{~cm}^{2} \mathrm{yr}\right)^{-1}$. It falls out from the common pattern, and we can suggest that we underestimate realistic number of events with that $F_{30}$, and they probably can be found in terrestrial isotope archives in future.

\section{Summary}

A new model of ${ }^{26} \mathrm{Al}$ production in lunar samples, in particular in rocks 64455,74275 and Apollo-15 deep core has been developed. With this model, we estimated the average GCR spectrum at the Myr time scale. The result modulation potential is $\phi=496 \pm 40 \mathrm{MV}$, and is close to $449 \pm$ $70 M V$ for the Holocene (the last 11000 years, Usoskin et al. (2016)). In contrast to that, this is significantly lower than one for the Modern Grand Maximum, (660 \pm 20 MV for 1951-2016, Usoskin (2017)). Because of the particular shape of the ${ }^{26} \mathrm{Al}$ yield function, it is possible to use nuclide data in a lunar sample at depths $<7 \mathrm{~g} / \mathrm{cm}^{2}$ as an integral particle spectrometer to evaluate the integral particle flux $F(>E)$. This was done for $20-80 \mathrm{MeV}$ particles. The result is in agreement with data from other nuclides for the last 11000 years, as well as with the data from space-borne 


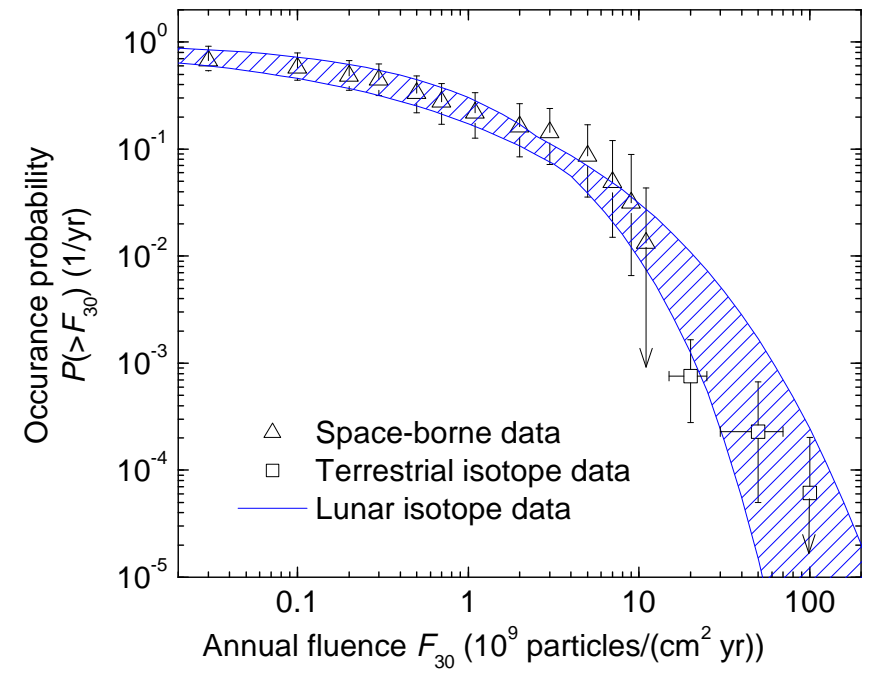

Figure 5: SEP occurrence probability density as a function of the annual fluence. The space-borne data is from (Usoskin and Kovaltsov, 2012), the terrestrial isotope data is modified after (Usoskin, 2017). The bars indicate $1-\sigma$ errors.

measurements for 1951-2008. Using the results of this work, we estimated the SEP occurrence probability distribution and compared it with estimates obtained from other data. It shows good agreement except one point. Its outlaying hints that we likely underestimate number of SEP events with $F_{30}=2 \times 10^{9}\left(\mathrm{~cm}^{2} \mathrm{yr}\right)^{-1}$. This provides new constrains on SEP events and can notably contribute to studies of the near-Earth's radiation environment.

\section{Acknowledgements}

Authors acknowledge the support from the Center of Excellence ReSoLVE (Project 272157) of the Academy of Finland, and they are thankful to Dr. Anton Artamonov for verification of simulations.

\section{References}

Agostinelli, S., Allison, J., Amako, K., et al. (2003). Geant4 - a simulation toolkit. Nucl. Instr. Meth. Phys. A, 506(3):250-303.

Fink, D., Klein, J., Middleton, R., Vogt, S., Herzog, G. F., and Reedy, R. C. (1998). ${ }^{41} \mathrm{Ca},{ }^{26} \mathrm{Al}$, and ${ }^{10} \mathrm{Be}$ in lunar basalt 74275 and ${ }^{10} \mathrm{Be}$ in the double drive tube 74002/74001. Geochimica et Cosmochimica Acta, 62:2389-2402.

Jull, A., Cloudt, S., Donahue, D., Sisterson, J., Reedy, R., and Masarik, J. (1998). 14c depth profiles in apollo 15 and 17 cores and lunar rock 68815. Geochimica et Cosmochimica Acta, 62(17):3025-3036.

Kovaltsov, G. A., Usoskin, I. G., Cliver, E. W., Dietrich, W. F., and Tylka, A. J. (2014). Fluence Ordering of Solar Energetic Proton Events Using Cosmogenic Radionuclide Data. Solar Phys., 289:4691-4700. 
Li, Y., Zhang, X., Dong, W., Ren, Z., Dong, T., and Xu, A. (2017). Simulation of the production rates of cosmogenic nuclides on the Moon based on Geant4. J. Geophys. Res. (Space Phys.), 122:1473-1486.

Meyer, C. (2012). Lunar Sample Compendium.

Nishiizumi, K., Arnold, J., Kohl, C., Caffee, M., Masarik, J., and Reedy, R. (2009). Solar cosmic ray records in lunar rock 64455. Geochimica et Cosmochimica Acta, 73(7):2163-2176.

Nishiizumi, K., Arnold, J. R., Klein, J., and Middleton, R. (1984). Al-26 depth profile in Apollo 15 drill core. Earth Planet. Sci. Lett., 70:164-168.

Poluianov, S. V., Kovaltsov, G. A., Mishev, A. L., and Usoskin, I. G. (2016). Production of cosmogenic isotopes ${ }^{7} \mathrm{Be},{ }^{10} \mathrm{Be},{ }^{14} \mathrm{C},{ }^{22} \mathrm{Na}$, and ${ }^{36} \mathrm{Cl}$ in the atmosphere: Altitudinal profiles of yield functions. Journal of Geophysical Research (Atmospheres), 121:8125-8136.

Rancitelli, L. A., Fruchter, J. S., Felix, W. D., Perkins, R. W., and Wogman, N. A. (1975). Cosmogenic isotope production in Apollo deep-core samples. In Lunar and Planetary Science Conference Proceedings, volume 6 of Lunar and Planetary Science Conference Proceedings, pages 1891-1899.

Reedy, R. C. (2007). Proton Cross Sections for Producing Cosmogenic Radionuclides. In Lunar and Planetary Science Conference, volume 38 of Lunar and Planetary Science Conference, page 1192.

Reedy, R. C. (2012). Update on Solar-Proton Fluxes During the Last Five Solar Activity Cycles. In Lunar and Planetary Science Conference, volume 43 of Lunar and Planetary Science Conference, page 1285 .

Reedy, R. C. (2013). Cosmogenic-nuclide production rates: Reaction cross section update. Nucl. Instr. Meth. Phys. B, 294:470-474.

Reedy, R. C. and Arnold, J. R. (1972). Interaction of solar and galactic cosmic-ray particles with the Moon. J. Geophys. Res., 77:537.

Usoskin, I. G. (2017). A history of solar activity over millennia. Living Reviews in Solar Physics, 14:3.

Usoskin, I. G., Gallet, Y., Lopes, F., Kovaltsov, G. A., and Hulot, G. (2016). Solar activity during the Holocene: the Hallstatt cycle and its consequence for grand minima and maxima. Astr. Astrophys., 587:A150.

Usoskin, I. G. and Kovaltsov, G. A. (2012). Occurrence of Extreme Solar Particle Events: Assessment from Historical Proxy Data. Astrophys. J., 757:92.

Vos, E. E. and Potgieter, M. S. (2015). New Modeling of Galactic Proton Modulation during the Minimum of Solar Cycle 23/24. Astrophys. J., 815:119. 